\title{
Thinking about thinking through inverse reasoning
}

\author{
Marlene Berke (marlene.berke@yale.edu), Julian Jara-Ettinger (julian.jara-ettinger@yale.edu) \\ Department of Psychology, Yale University
}

\begin{abstract}
Human Theory of Mind enables us to attribute mental states like beliefs and desires based on how other people act. However, in many social interactions (particularly ones that lack observable action), people also directly think about other people's thinking. Here we present a computational framework, Bayesian inverse reasoning, for thinking about other people's thoughts. Our framework formalizes inferences about thinking by inferring a generative model of reasoning decisions and computational processes, structured around a principle of $\mathrm{ra}$ tional mental effort - the idea that people expect other agents to allocate thinking rationally. We show that this model quantitatively predicts human judgements in a task where participants must infer the mental causes behind an agent's pauses as they navigate and solve a maze. Our results contribute to our understanding of the richness of the human ability to think about other minds, and to even think about thinking itself.
\end{abstract}

Keywords: Theory of Mind; Computational modeling; Social cognition

\section{Introduction}

Imagine playing chess and watching your opponent as they stare at the board. It's their turn, and they are taking a long time to move. There's an obvious move that they could make-they could take your queen with a pawn. If your opponent is a novice, you might think they just haven't noticed this option. But if your opponent is a Grandmaster, you might instead think that there's something more to it-maybe there's some downside to taking the queen, and you should try to figure out what it is. Or, maybe your opponent doesn't want to defeat you too quickly out of politeness, or maybe they aren't studying the board at all, but are daydreaming about things they could do over the holidays. Maybe they've even dozed off! If the Grandmaster at long last chooses a different move instead of taking your queen, you might suspect that they've noticed something that you didn't, and that you might be in for something worse than losing your queen.

The capacity to think about thinking is considered a cornerstone of Theory of Mind - the ability to interpret other people's behavior in terms of abstract mental states and processes (Wellman, 2014, Gopnik et al., 1997). Research in the last decade has begun to formalize the computations that underlie human Theory of Mind, showing that mental-state inference is structured around a principle of rational action - the idea that we expect agents to maximize the utilities they obtain while minimizing the costs they incur (Jara-Ettinger et al. 2020, Jern et al., 2017, Baker et al., 2017). This expectation is at work from early in infancy, enabling people to infer each other's knowledge, goals, and preferences (Lucas et al., 2014, Jara-Ettinger et al., 2016, Baker et al., 2009; Liu et al., 2017).

Yet, as the example above shows, reasoning about others' minds is not limited to mental-state attribution (determining what someone wants or knows), and also includes inferences about other people's mental processes (inferring what someone may or may not be thinking about). The apparent simplicity with which we make such inferences hides a complex computational problem. People's knowledge and preferences are often reflected in their behavior, such as watching an agent move in the direction of their goal. Yet, the mental computations that people perform are unobservable and often give little evidence beyond a lack of action.

Our goal in this paper is to introduce a computational framework that captures how people think about thinking. Modeling how we infer what other people are thinking about poses several theoretical challenges. What is our intuitive theory about how people decide what to think about? How do we expect agents' thinking to affect their behavior? And what is the connection between the amount of thinking an agent does and the amount of time that it takes them to think? Our framework, Bayesian inverse reasoning (BIR), proposes an answer to these questions, centered around a principle of $\mathrm{ra}$ tional mental effort. That is, we propose that people expect each other to use and allocate mental effort rationally, flexibly deciding when and what to think about as a function of what they intend to achieve. The principle of rational mental effort is analogous to the principle of rational action used in models of mental-state attribution, with the difference that it expresses assumptions about how agents use and allocate mental processes rather than assumptions about how agents move in space.

Research into mental effort has typically focused on firstperson situations, showing that people make rational tradeoffs between mental effort and accuracy (Shenhav et al., 2017; Kool \& Botvinick, 2018; Ongchoco et al., 2019). While the way people actually allocate effort does not necessarily need to match our intuitive theory of how people do this, some initial research suggests that, from childhood, we expect agents to use cognitive resources rationally (Liu et al. 2019) and that the amount of thinking is reflected in the time it takes agents to make choices (Richardson \& Keil, 2020). Our work draws inspiration from this qualitative research to propose a com- 
putational model of how we infer what others are thinking about.

The paper is structured as follows. We begin by introducing our computational framework and highlighting key similarities and differences from standard models of mental-state attribution. We then show how our model applies to a situation where observers watch an agent navigating a maze and must infer from their behavior the extent to which the agent is thinking about what to do. To elicit the graded inferences that our model supports, we vary the position and length of different pauses that the agent makes, and also vary the complexity of the decision the agent must make at different time points. We show that our model captures nuanced patterns of inferences that participants can make just by watching the agent's lack of action.

\section{Computational Framework}

Previous work suggests that human Theory of Mind can be modeled as Bayesian inference over a generative model that describes how mental states produce observable actions (JaraEttinger et al., 2020; Jara-Ettinger, 2019; Jern et al., 2017; Lucas et al., 2014). These previous models capture the idea that we expect agents to act so as to maximize the rewards that they obtain while minimizing the costs that they incur. Under this framework, mental-state attribution can be modeled as the process of inferring an agent's expected reward function given their choice of actions and the costs that these actions incur.

Our computational model takes Bayesian models of cognition as a starting point (see Tenenbaum et al. 2011 for an extended review of their use in modeling high-level cognition beyond Theory of Mind). The key difference from previous models is that our focus is not on inferring what an agent is doing, but inferring what an agent is thinking. Therefore, in contrast to previous models, our generative model is designed to capture our intuitive theory of what people think about, and our hypothesis space consists of computational processes, rather than reward functions associated with physical world states.

For simplicity and clarity, we present our model in the context of the task we use to evaluate its performance. Consider watching an agent solve a maze, having a bird's-eye view like the one shown in Figure 2 As the agent moves, suppose that they pause at a complicated intersection like the one in Figure $2 \mathrm{~B}$ indicated by the magenta dot. If the agent pauses there, it seems obvious that the agent must be thinking about what to do. But what if the intersection were a very simple one, like the one in Figure $2 \mathrm{C}$ indicated by the red $\mathrm{X}$ ? Then this pause will probably not seem like thinking to you. You might instead wonder whether the agent was thinking about something unrelated to the maze. And if the pause is at a place where there is little to think about, such as in the middle of a hallway, you might be confident that they were thinking about something else. Our model aims to capture these kinds of inferences.

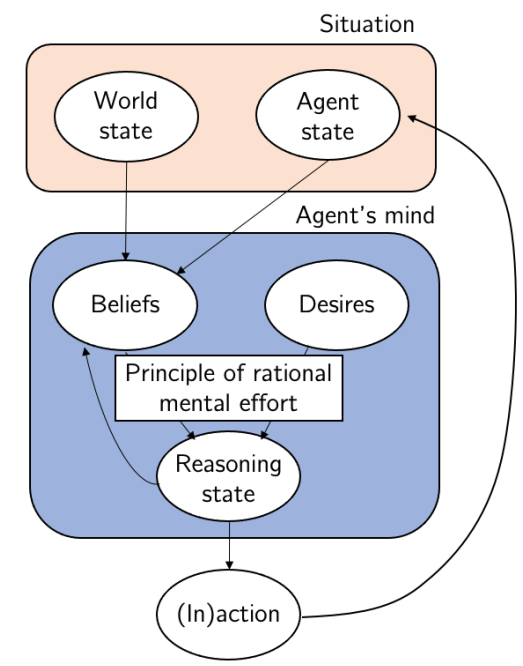

Figure 1: Conceptual model schematic. The yellow box represents the physical situation (the world state and the agent's physical state) and the blue box represents the agent's mind, which is hidden from observers.

We begin by presenting the generative model and inference procedure and then discuss our parameter choices and the logic behind them.

\section{Generative model}

Figure 1 shows a conceptual schematic of our model. In our framework, a situation is the combination of the world state (all physical information about the maze, consisting of a starting point, walls, and an exit) and the agent state (determining the agent's location within the maze), and we assume this information is observable and known. The core of the model is its description of the agent's beliefs, desires, and mental processes. In the context of our task, a desire consists of a simple goal to exit the maze (although this component can be easily extended to handle more complex desires like the ones developed in related work; Baker et al. 2017; Jara-Ettinger et al. 2020). In contrast to past frameworks that model beliefs as a probability distribution over world states, here we use a more nuanced representation that consists of a four-tuple $b=\{s, k, e, c\}$. These components capture four key representations that guide thinking: what situation am I in (state $s$ )? what do I know (knowledge $k$ )? what do I think I should do given what I know (value estimate $e$ )? and for how far ahead do I know what to do (certainty $c$ )? Formally, $s$ represents the situation (i.e., the world state and agent state); $k$ captures what the agent's knowledge, consisting of the subset of the maze that the agent has already analyzed (therefore including information about identified dead ends, and unexplored areas), represented as a tree over decisions; $e$ is the agent's estimate of value of different actions, based on what the agent knows. In this maze-solver model, $e$ describes the estimated value of moving toward different searched or partially searched branches of the maze. The value of moving 
toward a branch of the maze leading to a known dead end is 0 , the value of moving toward a branch known to lead to the exit point is $\operatorname{Inf}$, and the value of moving toward of a partially-searched branch is inversely related to the Manhattan distance between the agent's goal and the farthest known (already searched / thought about) point in that branch. The agent uses this assessment to guide its actions, choosing to move toward branches with high estimated values. Finally, $c$ is how far ahead the agent knows what to do, which we represent as the number of moves for which the agent already knows (based on previous searches) what decision to make.

A reasoning state then integrates the agent's beliefs and desires to decide whether to think or act. Here we focus on three types of reasoning states: deciding to act without further thought (henceforth acting), thinking directed at solving the maze (henceforth thinking), and thinking that is unrelated to the maze (henceforth distraction). Naturally, thinking and acting are not mutually exclusive, and our model includes a pervasive thinking component in which the agent is assumed to always be analyzing its surroundings, but can also decide to engage in a deeper analysis of the environment before deciding how to act.

The decision whether to think instantiates the principle of rational mental effort-the agent chooses to allocate its thinking when it expects thinking will be most useful in reducing its uncertainty about what to do next. Given that the agent's searches are depth-limited, it can only search a fixed number of moves ahead, $m s d$. If it already knows what to do for the next $c$ moves, then the utility of searching, or the number of moves ahead it could possibly learn about by thinking, is $m s d-c$. So, to maximize the expected utility of its searches, an ideal agent should think when $c$ is minimized, or when $c=0$.

Naturally, people don't expect agents to behave optimally all the time. We therefore relaxed the optimality assumption in our model to a probabilistic expectation that agents are more likely to do a deep search as a function of the utility of searching. Formally, the probability that the agent decides to think, based on how far ahead they know what to do $c$, is given by

$$
p(\text { think } \mid c)=\frac{1}{1+e^{f(c)}}
$$

where $f(c)$ is a linear model as a function of $c$ (see Parameter settings for details) and the full equation is thus a softmax function that indicates that agents are more likely to choose to think as the number of moves for which they know what to do decreases. This principle of rational mental effort instantiated in the generative model naturally creates an increased chance that an agent might choose to stop and think at intersections, or even a step before them, and is less likely to think after an intersection or in corridors with no upcoming intersections.

To model how an agent thinks about the maze we used a depth-limited depth first search (Korf], 1990), where possible routes that the agent can pursue are searched in a random order for a limited depth. This captures the idea that agents can scan paths to assess their value, but only do so for a limited amount of time (due to cognitive factors that we do not explicitly model, such as working memory load). The pervasive thinking aspect of our model (i.e., the assumption that the agent is always doing a rapid but short analysis of its surrounding) was modelled through a maximum search depth (msd) random variable sampled from a Poisson distribution $\operatorname{msd} \sim$ Poisson $\left(\lambda_{\text {pervasive }}\right)$. When the agent chooses to think about what to do next (Eq. 1), the lambda parameter for msd

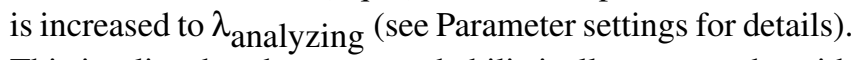
This implies that the agent probabilistically scans paths with different depths, and that, when it chooses to think, the search increases in depth significantly.

The final key component of the thinking process is the relationship between the amount of computation the agent does and how much time that thinking takes, capturing the intuitive notion that, in general, thinking more takes more time. To model this, we used a speed-of-thought factor that determined the amount of time it takes to analyze each square in milliseconds (see Parameter settings).

To model the possibility that the agent spontaneously thought about something unrelated to the task (distraction), which can happen at any point (including immediately before or after an agent has paused to think about the maze), we used an exponential distribution with parameter $\lambda_{\text {distraction }}$ that determined when and for how long the agent was distracted.

Altogether, the generative model specifies how an agent navigates a maze, monitors its surroundings, and decides when to engage in a deeper analysis of its surroundings to inform what to do next. At any point, a pause length is given by the amount of time it spends thinking plus the amount of time it spends distracted. Putting this all together, the generative model predicts that the length of time spent thinking at or before an intersection should depend on its complexity, a key prediction that we test in the experimental work.

\section{Inference}

Given a maze $m$ and a trajectory $\vec{a}$ (the locations and times spent at each step), we use the generative model to infer the amount of time spent distracted $t_{d}$ and the amount of time spent thinking $t_{t}$ given an observed pause of length $t$. We implemented the inference procedure via Markov Chain Monte Carlo (MCMC) using Metropolis-Hastings. For the smaller (7-by-7 and 10-by-10; see Experiment section for details) mazes, we conditioned the inference on the whole trajectory from the start of the maze to the finish, but for the large (15by-15) maze, conditioning on the entire path becomes a computationally difficult problem and so we conditioned inference only on the 20 locations before and after the pause of interest, as possible given the location of the pause (e.g., it is not possible to condition 20 steps after when the agent is only a few steps away from solving the maze). The MCMC chains were run for 500,000 iterations for the smaller mazes and 250,000 iterations for the large maze. The first half of the samples were removed as burn-in. 


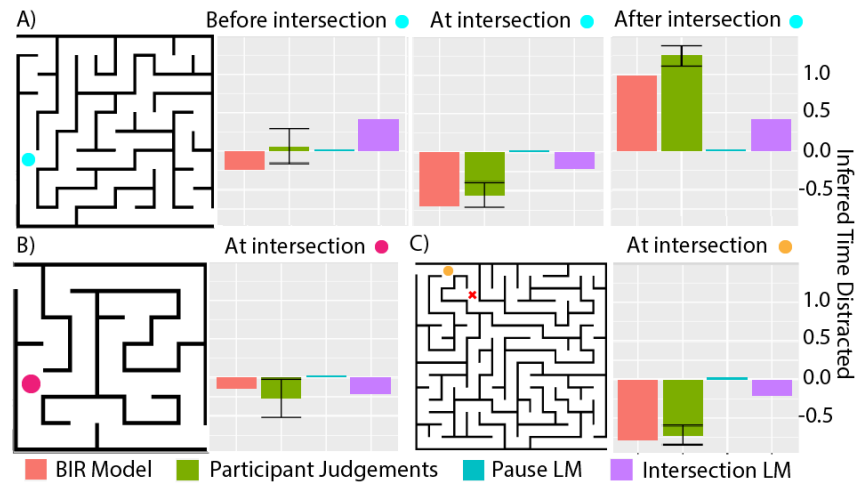

Figure 2: Three mazes used as stimuli in the experiment. The experiment used three different maze sizes (A-C) with pauses of different lengths at different positions in the maze, including intersections of varying complexity. (A) The three barplots on the top row show the results for a pause before (left barplot), at (middle barplot), and after (right barpot) the intersection labelled with the blue dot. (B) The leftmost barplot on the bottom row shows the results for a pause at the intersection labelled with a magenta dot on the maze to its left. (C) The rightmost barplot on the bottom row shows the results for a pause at the intersection labelled with an orange dot on the maze to its left. All of these pauses lasted 3.2 seconds. The $y$-axis on these barplots is the z-score: the more positive the z-score, the larger the portion of the pause attributed to distraction.

\section{Parameter settings}

Our computational framework requires that we set a number of critical parameters: the linear model that determines the softmax function $(f(c)$ in Eq. 1), the depth parameters for the agent's reasoning $\left(\lambda_{\text {pervasive }}\right.$ and $\left.\lambda_{\text {analyzing }}\right)$, the speedof-thought parameter, and the distribution of the length of distractions $\left(\lambda_{\text {distraction }}\right)$. Broadly speaking, there are two methods for setting these parameters. A first method is to fit these values to participant judgments, ensuring that they accurately capture participant's intuitions, but risking overfitting the data. A second method is to fix these parameters prior to data collection and show people an initial warm-up video that they can use to calibrate their expectations about how agents think in our task. Here we opted for the second method and therefore our experiment not only tests whether people can infer when other people are thinking about the task vs getting distracted, but the experiment also tests whether people have the flexibility to quickly adjust their expectations about how and when agents think from limited data.

All parameters were set prior to data collection as follows. The linear model within the softmax decision to think (Eq. 1) was set to $f(c)=-3+2.5 * c$ to ensure that the agent begins with a high probability of thinking when they don't know what to do next, and the probability of thinking rapidly decreases the more moves ahead that the agent is certain of what to do. The depth parameters for reasoning were set to $\lambda_{\text {pervasive }}=5$ truncated with bounds $[1,30]$, and $\lambda_{\text {reasoning }}=15$. The distribution of distraction times was set to $\lambda_{\text {distraction }}=0.02$ and the speed-of-thought was set to $100 \mathrm{~ms}$.

Having set these parameters, we also added a small amount of noise to each pause, sampled from a normal distribution $\sim \operatorname{Normal}(0, \sigma=100)$ (truncated at 0 and $100,000 \mathrm{~ms}$ ), included to make the inference procedure more tractable. Finally, the generative model is affected by the speed of the game, which we set to 600 milliseconds per move.

\section{Experiment}

Our computational model captures how agents decide when to think. This framework captures nuanced expectations that show a sensitivity not only to what information there is to think about, but also expectations about what is likely that agent might have already thought about as they were navigating, as well as how quickly they can think about different possibilities. Our Experiment is designed to test people's ability to derive these nuanced inferences by asking people to infer the extent to which a pause is due to thinking or distraction based on watching a short video of an agent navigating a maze. This experiment was pre-registered (link).

\section{Participants}

50 U.S. participants (mean age $=31.18$ years; range $=18-52$ years) were recruited from Prolific. An additional 11 participants were recruited but not included in the study based on pre-registered criteria, because they failed to pass the attention check questions ( $\mathrm{n}=11$; see Procedure).

\section{Stimuli}

Stimuli consisted of 23 short videos. Each video showed a bird's eye view of an agent navigating a maze for a few steps, pausing, and then moving for a few more steps. To elicit graded inferences, we used three different maze sizes that contained several intersections of varying complexity: a 7 by-7 maze ( $n=5)$ trials; Figure $2 \mathrm{~B})$, a 10-by-10 maze $(n=6)$; Figure 2A), and a 15-by-15 maze ( $\mathrm{n}=12)$; Figure $2 \mathrm{C})$. Each trial varied the location of the pause (esp. whether it was before, at, or after an intersection) and the duration of the pause. Additionally, one more 15-by-15 maze was used for a warm up, described in the Procedure section. The pre-registered set of all the stimuli are available at: (link).

\section{Procedure}

Participants first read a brief tutorial that explained that they would watch several videos of an agent pausing while solving a maze, and that their job would be to tell why the agent paused. For each video, participants were asked to indicate why the agent paused using a slider from "thinking for the whole pause" (coded as 0) to "distracted for the whole pause" (coded as 100), with the midpoint labeled "thinking for the half, distracted for half" (coded as 50). 
Participants had to pass three simple attention checks to ensure that they had read the instructions before they could continue into the experiment. Incorrect responses for any attention check resulted in expulsion from the experiment. After an introduction that explained that the agents in this experiment pause because of distraction and/or thinking, Attention Check 1 asked participants to select from a list of options all of the possible reasons why the agent might pause. Attention Check 2 asked participants to place the slider where it should go if they know that the agent was distracted for the whole pause. Slider response values of 90 or greater were considered correct. Attention Check 3 followed the warm up video (described below), whose introduction stipulated that in this video, pauses are only ever due to thinking. Slider response values of 10 or less were considered correct.

To give the participants an idea of the speed at which the agent can move and think, participants first watched a warm up video of an agent solving a maze (which was not used in the experimental task). This video included 4 pauses, all at intersections, showing the mode (single most likely) pause duration. The participants were told that, in this video, all of the person's pauses were related to thinking rather than distraction. Thus, this video allowed participants to understand the speed of the game and get an intuition about the agent's speed of thought, without revealing too much information about the task (critically, participants never saw the contribution of distractions or the variability in the distribution of thinking pauses).

After passing the attention checks, participants watched and responded to all 23 videos presented in random order.

\section{Results}

To compare the model's predictions to participant judgements, we first z-scored responses within participants, then averaged those responses for each stimulus. We also z-scored the proportion of the pause's duration that the model attributed to distraction. The correlation between participant judgements and model predictions was $r=0.80$, with a $95 \%$ confidence interval $(0.58,0.91)$. Figure 3 show the results of the experiment, revealing how the model was able to capture not only a qualitative distinction between thinking and distraction, but also graded quantitative estimates of how much time an agent spent thinking or distracted. The high correlation between the model and participants is surprising, particularly given the computational complexity of this problem, the fact that the data consisted of a single short pause on different parts of a maze, and the fact that the model's parameters were not fit to participant data, but selected ahead of time. To show that the generative model explains participant responses above and beyond what a cue-based model could do, we also performed a linear regression using pause time (Pause LM), finding a correlation of $r=0.069$. A linear regression of participant responses against whether or not the pause was located at an intersection (Intersection LM) produced a correlation of $r=0.495$. A third linear regression including pause time, whether or not the pause occurred at an

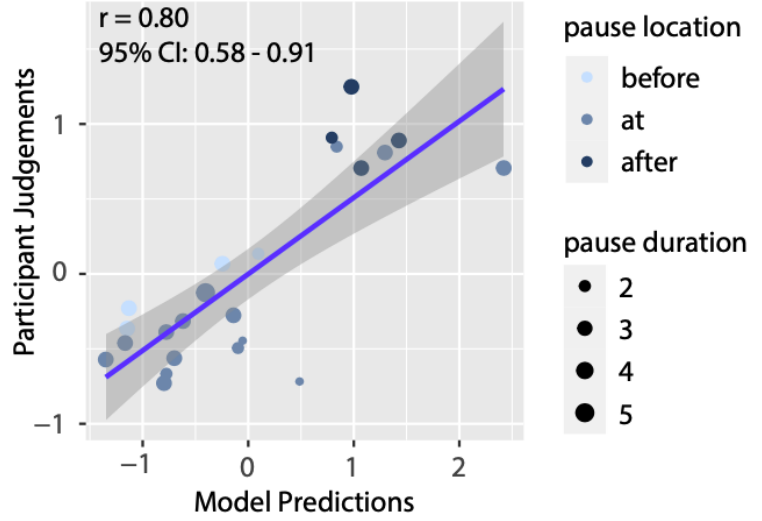

Figure 3: Experiment results. Each point represents a trial with model prediction on the $\mathrm{x}$ axis and participant judgment on the $y$ axis. The blue line shows best linear fit between the model and the data. The hues of the points represent the location of the pause relative to the nearest intersection, and the sizes represent the duration of the pause, in seconds.

intersection, and the interaction between those two predictors produced a correlation of $r=0.506$.

Figure 2 shows the results of five experiment trials, that highlight the nuance in people's inferences, along with predictions from our Bayesian inverse reasoning (BIR) model and the two alternative cue-based models. A key prediction of the BIR model is that pauses driven by thinking should occur at or (with lower probability) before intersections and are unlikely to occur after intersections. The top row of barplots in Figure 2A illustrates that participant judgements (green bar) follow this predicted pattern; the pause occurring at the intersection (middle barplot) was inferred to be because of thinking, while the pause after the intersection (right barplot) was inferred to be because of a distraction, and the pause before the intersection (left barplot) was ambiguous.

Another key prediction of the BIR model is that participant judgments about how much an agent was thinking should be sensitive to the complexity of the intersection at which they paused. To see the effects of intersection complexity on participant judgements and on model predictions, compare the three barplots in Figure 2 corresponding to pauses at the simple (magenta), and complex (blue and orange) intersections (magenta: bottom row, left barplot, blue: top row, middle barplot, and orange: bottom row, right barplot). Both participant judgements and the BIR model's predictions favor thinking as the complexity of the intersection increases, while neither the Intersection nor Pause LMs show this behavior.

Qualitatively examining the largest discrepancies are useful for assessing why the model failed in certain cases. The largest discrepancy between model predictions and human judgements occurred for a 3.2 second pause on the maze shown in Figure 2 $\mathrm{C}$ at the very simple intersection marked with a red $\mathrm{X}$. This trial corresponds to the rightmost point in 
Figure 3 At this simple intersection, both participants and the model inferred that distraction caused most of the agent's pause, but the model predicted that pause was wholly due to distraction, and that thinking played no role. A preliminary analysis suggests our MCMC algorithm failed to obtain highprobability samples, which could be solved by using a better proposal function.

Another large discrepancy occurred for a 1.6 second pause on the maze shown in Figure $2 \mathrm{C}$ at the complex intersection marked with an orange dot. This trial corresponds to the point in Figure 3 at roughly $(\mathrm{x}=0.5, \mathrm{y}=-0.75)$. A qualitative analysis of this error suggests that the poor fit was due to the model's very tight prior on the speed of thought, as our model inferred that the timing was too short for the agent to have had the opportunity to analyze the scene. Participants likely don't share such a tight prior over the speed of thought, making them more likely to explain the pause by thinking.

\section{Discussion}

Here we presented the Bayesian inverse reasoning framework for modeling how we think about another agent's thinking. Our model reasons about other people's mental processes as Bayesian inference over a generative model of how agents decide when to think, structured around a principle of rational mental effort - the expectation that agents allocate and use computational resources rationally. We tested our framework in the context of a maze navigation task where participants had to infer whether an agent's inaction was attributable to thinking or distraction or both. Our model predicted participant judgments with quantitative accuracy, showing that people do not make these judgements purely based on simple strategies. Instead, participants displayed sensitivity to the position of the pause, its length, and the complexity of upcoming intersections, as our model predicted.

As shown in Figure 3, participant judgements and model inferences seem to form two clusters: one where the agent was judged to be thinking, and another where the agent was judged to be distracted. We believe that this is an artifact of a lack of ambiguous videos in the stimulus set. In any case, both the participants and the model display this clustering, providing further support for our model. In future work we plan to test the model in ambiguous cases.

Our work contrasts with previous models of Theory of Mind by modeling how people perform inference over mental processes rather than mental states. This required us to build richer mental state representations than previous work has used. Our work therefore also provides a first step toward the development of richer representations of others' beliefs, which include not only knowledge about the state of the world, but also an awareness of how much knowledge they have and how much more they need to know to satisfy their desires.

All parameters in our model were set prior to collecting any data. Rather than fit these parameters to participant data, we instead tried to provide participants with these priors by showing them a warm up video where pauses lasted the mode duration suggested by the model. The fact that participant data aligned well with model predictions implies that, without any information about the variance of the distributions used in the generative model, participants were still able to learn these priors from very little data. This suggests that people possess an impressive ability to infer not only what other agents are thinking about, but also the relationship between thinking and behavior for an agent.

Our Bayesian inverse thought framework posits a principle of rational effort: the idea that people expect other agents to allocate mental effort (thinking) in an efficient and rational manner. A key component of this framework is a four element tuple $b$, representing the beliefs necessary to inform allocation of thinking. To deploy thought in a rational manner for solving a task, the agent should represent the state of the world $s$, the state of the agent's own knowledge $k$, value estimates $e$ of possible actions, and and of how many upcoming actions $c$ the agent is certain. This tuple of representations underpins the principle of rational effort, and we expect this to be fruitful for modeling inferences about an agent's thoughts in a broad a variety of tasks. We believe that this framework captures key elements of people's intuitive theory of how other agents think.

Within this framework, there are other formulations of the model that might be fruitful to explore in future work. For example, the optimal policy for when to think or not may change based on the formulation of the problem. Here, we formulated the problem as maximizing the utility of thinking. But it could have been formulated in other ways. For instance, optimizing for solving the maze as quickly as possible vs as flawlessly as possible (risking no mistakes and backtracking) may lead to different optimal policies. We expect to explore other models within this Bayesian inverse thought framework in future work.

Altogether, this work advances our understanding of the computations behind people's ability to think about other people's thinking, and it highlights people's capacity to make rich mentalistic inferences from the simplest behaviors, such as a short pause between otherwise smooth movements.

\section{Acknowledgments}

We thank Emory Richardson for helpful discussions. This work was supported by a Google Faculty Research Award and by the Center for Brains, Minds, and Machines NSF-STC award CCF-1231216.

\section{References}

Baker, C. L., Jara-Ettinger, J., Saxe, R., \& Tenenbaum, J. B. (2017). Rational quantitative attribution of beliefs, desires and percepts in human mentalizing. Nature Human Behaviour, 1(4), 0064.

Baker, C. L., Saxe, R., \& Tenenbaum, J. B. (2009). Action understanding as inverse planning. Cognition. 
Gopnik, A., Meltzoff, A. N., \& Bryant, P. (1997). Words, thoughts, and theories. Mit Press Cambridge, MA.

Jara-Ettinger, J. (2019). Theory of mind as inverse reinforcement learning. Current Opinion in Behavioral Sciences.

Jara-Ettinger, J., Gweon, H., Schulz, L. E., \& Tenenbaum, J. B. (2016). The naïve utility calculus: Computational principles underlying commonsense psychology. Trends in cognitive sciences, 20(8), 589-604.

Jara-Ettinger, J., Schulz, L. E., \& Tenenbaum, J. B. (2020). The naive utility calculus as a unified, quantitative framework for action understanding. Cognitive Psychology.

Jern, A., Lucas, C. G., \& Kemp, C. (2017). People learn other people's preferences through inverse decisionmaking. Cognition, 168, 46-64.

Kool, W., \& Botvinick, M. (2018). Mental labour. Nature human behaviour, 2(12), 899-908.

Korf, R. E. (1990). Depth-limited search for real-time problem solving. Real-Time Systems, 2(1-2), 7-24.

Liu, S., Cushman, F., Gershman, S., Kool, W., \& Spelke, E. S. (2019). Hard choices: Children's understanding of the cost of action selection. In Cogsci (pp. 671-6677).

Liu, S., Ullman, T. D., Tenenbaum, J. B., \& Spelke, E. S. (2017). Ten-month-old infants infer the value of goals from the costs of actions. Science, 358(6366), 1038-1041.

Lucas, C. G., Griffiths, T. L., Xu, F., Fawcett, C., Gopnik, A., Kushnir, T., ... Hu, J. (2014). The child as econometrician: A rational model of preference understanding in children. PloS one, 9(3), e92160.

Ongchoco, J. D., Jara-Ettinger, J., \& Knobe, J. (2019). Imagining the good: An offline tendency to simulate good options even when no decision has to be made..

Richardson, E., \& Keil, F. (2020). Children use agents' response time to distinguish between memory and novel inference.

Shenhav, A., Musslick, S., Lieder, F., Kool, W., Griffiths, T. L., Cohen, J. D., \& Botvinick, M. M. (2017). Toward a rational and mechanistic account of mental effort. Аnпиal review of neuroscience, 40, 99-124.

Tenenbaum, J. B., Kemp, C., Griffiths, T. L., \& Goodman, N. D. (2011). How to grow a mind: Statistics, structure, and abstraction. science, 331(6022), 1279-1285.

Wellman, H. M. (2014). Making minds: How theory of mind develops. Oxford University Press. 